\title{
CME: Hepatology (132431): self-assessment questionnaire
}

\author{
Authors: Edited by Sushma Saksena, Abhishek Chauhan and Tahseen A Chowdhury
}

\section{SAQs and answers are ONLINE for RCP fellows and collegiate members}

\section{Format}

Candidates are asked to choose the best answer from the five possible answers. This best of five format is used in many medical examinations; however, the questions are not intended to be representative of those used in the MRCP(UK) Part 1 or Part 2 Written Examinations.

\section{The answering process}

1 Go to www.rcplondon.ac.uk/SAQ

2 Log on using your usual RCP username and password

3 Select the relevant CME question paper

4 Answer all 10 questions by selecting the best answer from the options provided

5 Once you have answered all the questions, click on Submit

\section{Registering your external CPD credits}

Carrying out this activity allows you to claim two external CPD credits. These will be automatically transferred to your CPD diary, where you can review the activity and claim your points.

1. Which of the following patients with acute liver injury is LEAST likely to resolve with medical treatment alone?

(a) Acute fatty liver of pregnancy.

(b) Autoimmune hepatitis.

(c) Paracetamol overdose.

(d) Seronegative hepatitis.

(e) Viral hepatitis.

2. A 54-year-old man of South Asian ethnicity with type 2 diabetes and hypertension was referred to the hepatology clinic with deranged liver function tests (serum alanine aminotransferase (ALT) at $58 \mathrm{U} / \mathrm{L}$ ). He does not drink alcohol and his liver aetiology screen was negative for viral hepatitis, autoimmune or metabolic liver diseases and malignancy. He takes ramipril, metformin and gliclazide and no other prescribed or non-prescribed substances. Liver ultrasound demonstrated bright echotexture. Based on his ALT, aspartate aminotransferase ( $49 \mathrm{U} / \mathrm{L})$ and platelet count $\left(203 \times 10^{9} / \mathrm{L}\right)$, the fibrosis- 4 index for liver fibrosis (Fib-4) score is indeterminate and he is referred for transient elastography (FibroScan) which shows a liver stiffness of $9.0 \mathrm{kPa}$

Which of the following is the next stage in his management?

(a) Bariatric surgery.

(b) Behavioural and lifestyle counselling.

(c) Obeticholic acid.

(d) Pioglitazone.

(e) Vitamin E.

\section{Which one of following statements is true?}
(a) Ammonia promotes muscle protein synthesis.
(b) Hand grip strength is a worse predictor of adverse clinical outcomes than computed tomography measures of muscle.
(c) Patients with sarcopenia should avoid prolonged fasting.
(d) Sarcopenia is an irreversible condition.
(e) Sarcopenia is more prevalent in compensated cirrhosis than decompensated cirrhosis.

4. What is the recommended daily protein requirement in decompensated cirrhosis?
(a) $0.5-1.0 \mathrm{~g} / \mathrm{kg}$.
(b) $1.0-2.0 \mathrm{~g} / \mathrm{kg}$.
(c) $1.2-1.5 \mathrm{~g} / \mathrm{kg}$.
(d) $1.5-2.0 \mathrm{~g} / \mathrm{kg}$.
(e) $2.0-3.0 \mathrm{~g} / \mathrm{kg}$.

5. A 45-year-old man with history of alcohol-related cirrhosis diagnosed 6 months previously was admitted due to fever, new onset ascites and poor general condition. At admission, he was noted to have mean arterial pressure of $83 \mathrm{mmHg}$, saturated oxygen at $96 \%$ on room air, hepatic encephalopathy grade 2, jaundice and grade 3 ascites. His serum bilirubin was $376 \mu \mathrm{mol} / \mathrm{L}$, international normalised ratio of 3.2, serum creatinine at $106 \mu \mathrm{mol} / \mathrm{L}$, leukocytes count of $15.4 \times 10^{3} / \mathrm{mL}$, serum sodium of $125 \mathrm{mEq} / \mathrm{L}$, ascitic fluid polymorphonuclear cells at 2,160 cells $/ \mathrm{mm}^{3}$.

\section{What is the diagnosis?}
(a) Acute decompensation.
(b) Acute-on-chronic liver failure grade 1.
(c) Acute-on-chronic liver failure grade 2.
(d) Acute-on-chronic liver failure grade 3.
(e) Advanced decompensated liver cirrhosis. 
6. Which one of the following terms describes the process aimed at analysing the assortment of expressed ribonucleic acids by high-throughput sequencing of the corresponding copy deoxyribonucleic acids (cDNAs)?
(a) Metabolomics.
(b) Metagenomics.
(c) Metaproteomics.
(d) Metataxonomics.
(e) Metatranscriptomics.

7. A 56-year-old man, previously managed as an outpatient, with compensated cirrhosis due to metabolic-dysfunctionassociated liver disease, in conjunction with type 2 diabetes and hypertension, was admitted to hospital for the first time with haematemesis and ascites. He was a participant in a study evaluating the gut microbiota in patients with cirrhosis. His haemoglobin was $96 \mathrm{~g} / \mathrm{L}$ (115-165), creatinine was $130 \mu \mathrm{mol} / \mathrm{L}(60-110)$, total bilirubin $58 \mu \mathrm{mol} / \mathrm{L}$ (3-17), prothrombin time 18 seconds (11.5-15.5), upper gastrointestinal endoscopy had three columns of oesophageal varices - band ligation, and diagnostic paracentesis had ascites neutrophil count $>250 / \mathrm{mm}^{3}$.

Comparing the gut microbiota prior to his admission, which bacterial species is most likely to have increased in relative abundance?
(a) Bacteroidaceae.
(b) Clostridiales XIV.
(c) Enterococcaceae.
(d) Lachnospiraceae
(e) Veillonellaceae.

8. A 47-year-old woman presented with chronic fatigue and her general practitioner had noted abnormal liver function tests for the previous 8 months. Her blood results were alkaline phosphatase $311 \mathrm{IU} / \mathrm{L}$ (normal range $<130$ ), alanine aminotransferase $110 \mathrm{U} / \mathrm{L}$ (normal $<40$ ), bilirubin $11 \mu \mathrm{mol} / \mathrm{L}$ (normal $<21$ ), antinuclear antibodies 1:160, antimitochondrial antibodies 1:80 (M2 subunit positive). Her liver ultrasound was normal.

\section{What is the most likely diagnosis?}

(a) Cholestatic drug reaction.

(b) Intra-hepatic cholangiocarcinoma.

(c) Primary biliary cholangitis.

(d) Primary biliary cholangitis - autoimmune hepatitis overlap syndrome.

(e) Primary sclerosing cholangitis.
9. A 32-year-old man with a recent diagnosis of primary sclerosing cholangitis presented to his general practitioner (GP) with a 2-week history of worsening pruritus and new onset jaundice. He had also complained of anorexia, $2 \mathrm{~kg}$ weight loss and low-grade fever. His GP noted an elevated bilirubin of $123 \mu \mathrm{mol} / \mathrm{L}$ (normal range $<21$ ) and an abnormal CA $19-9$ of $235 \mathrm{U} / \mathrm{mL}$ (normal $<37 \mathrm{U} / \mathrm{mL}$ ). He was referred to secondary care.

\section{What is the most appropriate diagnostic test?}

(a) Computed tomography of the liver with contrast.

(b) Endoscopic retrograde cholangiopancreatography.

(c) Magnetic resonance imaging of the liver with contrast and magnetic resonance cholangiopancreatography.

(d) Percutaneous ultrasound-guided liver biopsy.

(e) Ultrasound of the liver.

10. A 56 -year-old woman (weighing $84 \mathrm{~kg}$ ) with known diagnosis of primary biliary cholangitis attended for her regular outpatient clinic review. She had been taking ursodeoxycholic acid (UDCA) $750 \mathrm{mg} /$ day for the last 2 years with good compliance. She complained of pruritis and her clinic blood tests showed bilirubin at $13 \mu \mathrm{mol} / \mathrm{L}$ (normal range $<21$ ) and alkaline phosphatase at 272 IU/L (normal range $<130$ ).

\section{What is the most important change to her therapy?}
(a) Add in bezafibrate $200 \mathrm{mg}$ per day.
(b) Add in obeticholic acid $5 \mathrm{mg}$ per day.
(c) Increase UDCA to $1,000 \mathrm{mg}$ per day.
(d) Increase UDCA to $1,250 \mathrm{mg}$ per day.
(e) Prescribe chlorpheniramine $4 \mathrm{mg}$ when required.

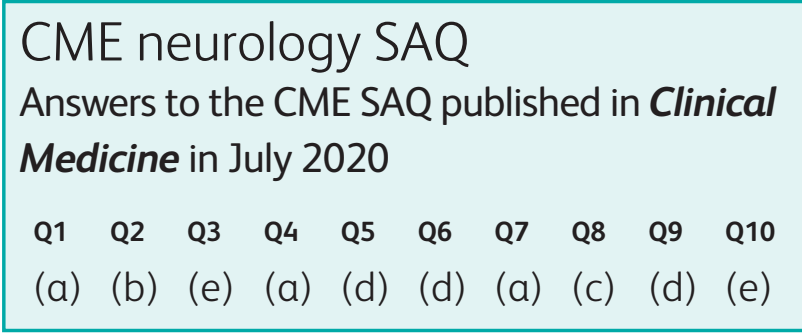

